\title{
Program Analysis via Satisfiability Modulo Path Programs
}

\author{
William R. Harris ${ }^{1}$, Sriram Sankaranarayanan ${ }^{2}$, Franjo Ivančić ${ }^{3}$ and Aarti Gupta ${ }^{3}$ \\ 1. University of Wisconsin, 2. University of Colorado, Boulder, 3. NEC Laboratories America, Princeton. \\ wrharris@cs.wisc.edu, srirams@colorado.edu, \{ivancic,agupta\}@nec-labs.com
}

\begin{abstract}
Path-sensitivity is often a crucial requirement for verifying safety properties of programs. As it is infeasible to enumerate and analyze each path individually, analyses compromise by soundly merging information about executions along multiple paths. However, this frequently results in a loss of precision. We present a program analysis technique that we call Satisfiability Modulo Path Programs (SMPP), based on a path-based decomposition of a program. It is inspired by insights that have driven the development of modern SMT (Satisfiability Modulo Theory) solvers. SMPP symbolically enumerates path programs using a SAT formula over control edges in the program. Each enumerated path program is verified using an oracle, such as abstract interpretation or symbolic execution, to either find a proof of correctness or report a potential violation. If a proof is found, then SMPP extracts a sufficient set of control edges and corresponding interference edges, as a form of proof-based learning. Blocking clauses derived from these edges are added back to the SAT formula to avoid enumeration of other path programs guaranteed to be correct, thereby improving performance and scalability. We have applied SMPP in the F-Soft program verification framework, to verify properties of real-world C programs that require path-sensitive reasoning. Our results indicate that the precision from analyzing individual path programs, combined with their efficient enumeration by SMPP, can prove properties as well as indicate potential violations in the large.
\end{abstract}

\section{Categories and Subject Descriptors}

D.2.4 [Software/Program Verification]: Assertion checkers; F.3.1 [Specifying and Verifying and Reasoning about Programs]: Assertions; F.3.2 [Semantics of Programming Languages]: Program analysis

\section{General Terms}

Languages, Verification

Permission to make digital or hard copies of all or part of this work for personal or classroom use is granted without fee provided that copies are not made or distributed for profit or commercial advantage and that copies bear this notice and the full citation on the first page. To copy otherwise, to republish, to post on servers or to redistribute to lists, requires prior specific permission and/or a fee.

POPL'10, January 17-23, 2010, Madrid, Spain.

Copyright 2010 ACM 978-1-60558-479-9/10/01 ...\$10.00.

\section{INTRODUCTION}

Path-sensitivity is often a crucial requirement for verifying safety properties of programs. As it is infeasible to enumerate and analyze each path individually, analyses compromise by soundly merging information about executions along multiple paths. However, this results in a loss of precision, which may lead the analysis to determine falsely that a violation is possible. We present the Satisfiability Modulo Path Programs (SMPP) approach to program analysis for property verification. Our approach decomposes the verification of a given program to verification of its component path programs [4]. A path program represents a set of program executions, all of which traverse the same set of edges in a control flow graph, but may vary in the number of iterations of loops/recurrences encountered. Each path program in a given Control Flow Graph (CFG) is associated with a simple path in the MSCC (Maximal Strongly Connected Component) decomposition of the CFG, obtained by compacting loops and recurrences in the program into components [9]. Whereas the number of control paths in the original CFG may be infinite (due to loops and recurrences), the MSCC decomposition is acyclic with finitely may control paths. Nevertheless, this number can be prohibitively large for real-world programs. In our experiments, we have observed millions of path programs even for moderate-sized CFGs with about 500 control edges. Thus an explicit enumeration of path programs is not feasible.

We therefore propose a SAT-based symbolic encoding of the set of control paths associated with path programs. Starting from a SAT formula that represents all unexplored control paths in the MSCC graph that can reach an error location, we enumerate a control path and use an oracle to verify the corresponding path program. Each enumerated path program can be analyzed using "proof techniques" such as abstract interpretation $[12,11]$, or "falsification techniques" such as bounded model-checking that search for concrete error traces of violations [5]. We assume (w.l.o.g.) that a verification oracle presents Floyd-Hoare style proofs in the form of inductive invariants, or concrete witnesses upon violation.

We present a proof-based learning technique that avoids enumerating a large set of "related" path programs by reusing the proof of a single path program. The proof-based learning technique extracts a set of sufficient edges from a given path program and an associated set of interference edges. Our technique guarantees that any other path program that also traverses the same set of sufficient edges and none of the interference edges is also correct. As a result, our ap- 
proach extends the core reasons for the proof of a given path program to apply to a large number of closely related path programs. The sufficient and interference edges are encoded as blocking clauses and added back to our SAT formula. We continue to enumerate solutions to this SAT formula until no more solutions can be found. Using proof-based learning significantly reduces the total number of path programs enumerated in SMPP, in the most dramatic case from millions to hundreds.

Our SAT encoding can be viewed as a control-flow abstraction with refinement performed by proof-based learning of sufficient/interference edges in the CFG. We do not encode or directly abstract data values. Reasoning over data is performed by the verification oracles. Note also that our enumeration operates over path programs and not program traces.

We present an instantiation of our approach that utilizes the abstract interpretation framework as the oracle of choice to prove properties over path programs. The inductive invariants obtained through abstract interpretation are used to extract sets of sufficient edges. We also present the use of over-approximate symbolic execution as another oracle that is efficient over path programs without loops or wherein the property to be proved is independent of the loops. It is possible to use other known techniques as oracles, such as lazy abstraction with interpolants [26] or predicate abstraction refinement $[17,2,3]$.

We implemented the SMPP technique in the F-Soft program verification framework [21]. The implementation uses a symbolic execution engine based on Yices [16], and abstract interpretation engines using a succession of numerical domains - intervals [10], octagons [27], symbolic intervals [30] and polyhedra [13]. We evaluated our implementation by using it to verify array overflow and string library usage properties for $\mathrm{C}$ programs. We used publicly available benchmarks - smaller programs in Zitser et al. [34], and larger open source programs such as openssh, thttpd and xvidcore. Our evaluation shows that SMPP can derive proofs of properties that are beyond the reach of pathinsensitive static analysis (already implemented in F-Soft), and it also identifies numerous potential violations. In comparison to the BLAST tool (using predicate abstraction with interpolant-based refinement [3]), SMPP can prove a majority of the properties proved by BLAST and identify additional violations in these programs, within a fraction of the time taken by BLAST.

Analogy with SMT Solvers: To prove safety properties, SMPP integrates program analysis oracles that prove properties about individual path programs with a SAT solver, which is used to enumerate over a Boolean abstraction of the control-flow of the program. This is similar in spirit to Satisfiability Modulo Theory (SMT) solvers [29, 16, 15]. To check the satisfiability of a given formula, an SMT solver integrates theory solvers (that check the satisfiability of conjunctive formulas over a theory) with a SAT solver that enumerates over a Boolean abstraction of the entire formula.

To solve a particular conjunctive formula, an SMT procedure employs theory solvers as oracles. Similarly, SMPP uses verification techniques over path programs as oracles. In fact, we may use different oracles for different path programs, based on observed characteristics (e.g. whether or not a backward slice w.r.t the property contains loops). Furthermore, the characteristics of the oracles affect the char-

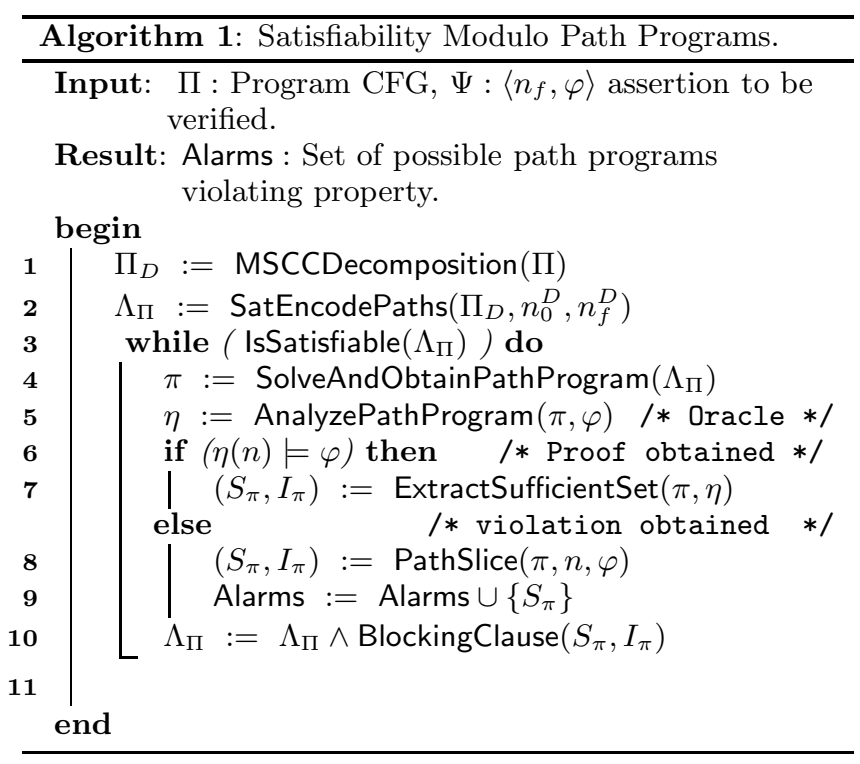

acteristics of our overall scheme (soundness, completeness, performance, etc.).

Theory-based learning in SMT solvers adds conflict clauses back to the SAT solver to refine the Boolean abstraction. Analogously, SMPP utilizes proof-based reasoning to derive sufficient edges and interference edges. These are added as blocking clauses to a SAT formula to block other enumerations by the SAT solver. By relying on a Boolean formula abstraction, we can apply SAT-based decision heuristics and blocking clauses that can drive the symbolic search over control paths in a property-driven manner. This is potentially advantageous compared to an a priori fixed search order on the CFG such as depth-first search (as in typical symbolic execution based approaches) or breadth-first search (as in typical BMC-based approaches).

The main contributions of this paper are: (a) SMPP: An approach for symbolic enumeration of path programs, using a SAT solver and proof-generating oracles for verifying path programs. (b) Proof-based learning techniques to identify and re-use a set of sufficient edges that serve as proofs of correctness. (c) A concrete instantiation of the SMPP approach, where we use abstract interpretation over different abstract domains and symbolic execution as oracles. (d) An experimental evaluation of the implementation, which shows that SMPP can prove more properties than path-insensitive static analysis and improve performance over predicate abstraction refinement. In particular, SMPP avoids the cost of an expensive data abstraction-refinement process and divergences on loops, while fully utilizing advances in SAT/SMT solvers.

\subsection{SMPP Approach At a Glance}

Algorithm 1 presents the main steps of the SMPP algorithm. We now step through it with an example. Fig. 1 presents a simple imperative program fragment that computes a buffer length bLen based on an input pointer $\mathrm{p}$, its length pLen, and an extra flag mode. The CFG representation is also given (also Cf. Example 2.1). The goal is to prove the unreachability of the CFG node labeled 15 corresponding to the program assertion at line 15. Fig. 2 depicts 

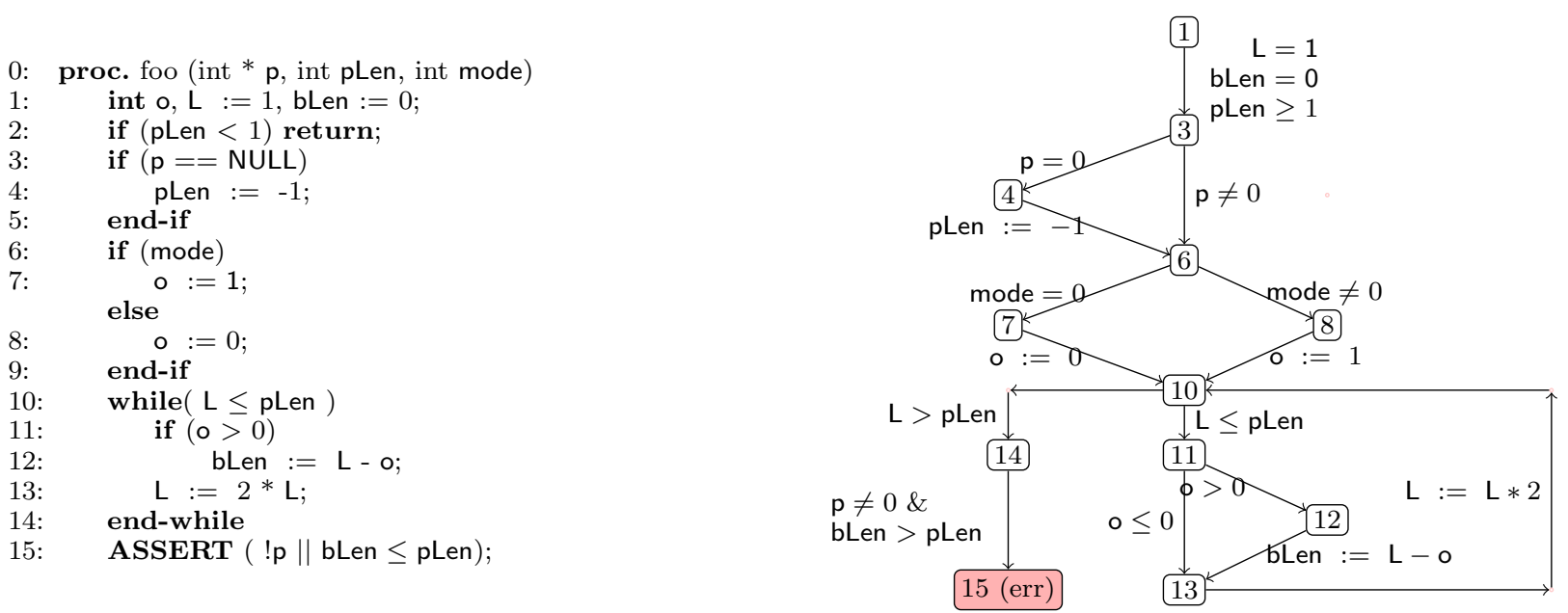

Figure 1: A simple imperative program and its CFG representation.

the major steps in the application of SMPP to the program, described as follows.

Extract an unexplored path program: SMPP first constructs the MSCC decomposition $\Pi_{D}$ of the program and encodes the set of all paths in $\Pi_{D}$ (Algo. 1 lines $\left.1-2\right)$. It then chooses an unexplored path from this set (line 4), from which it constructs path program (A), illustrated as the path program highlighted in Fig. 2(A). Considering just the nodes and edges that lie on this path program, the unreachability of 15 can be proved by a Constants-domain analysis (Algo. 1 lines 5-6).

Extract a set of sufficient edges: The proof of unreachability of node 15 for path program (A) involves the invariant $p=0$ that is valid at node 13 along the path program (A) (the invariant does not, in fact, hold for the program as a whole). SMPP applies a technique (detailed in Sec. 3.2) that pinpoints the key "reason" for the invariant. Such a "reason" takes the form of a set of sufficient (control-flow) edges. The set of sufficient edges (denoted $S_{1}$ ) corresponding to the proof in the first path program is shown in Fig. 2(B).

Any path program from 1 to 15 that traverses all of the edges in set $S_{1}$ will satisfy the property. Note that this includes paths that traverse the loops in the program arbitrarily many times as well. Computing sufficient sets therefore allows us to extend our proof along a single path program to multiple path programs. This step corresponds to Algo. 1 line 7 .

Extract another path program: SMPP now seeks a path program that visits edges $E$ such that $S_{1} \nsubseteq E$. The search for such a path program is performed using a SAT solver (Algo. 1 lines 10,3), and yields the second path program, as shown in Fig. 2(C). Now, the analysis must directly reason about the loop in this path program to prove unreachability of node 15 . It can do so automatically by using the octagon domain analyzer [27] to establish the invariant bLen $\leq$ pLen at node 14 (Algo. 1 lines 5-6). This invariant proves correctness of this path program. The loop invariant bLen $\leq$ pLen computed at node 10 suffices to prove the property at node 15 . Note that this invariant is independent of the variable $\mathrm{o}$.
SMPP then extracts a set of sufficient edges $S_{2}$ for the invariant (detailed in Sec. 3.2), shown in Fig. 2(D). It also performs an interference analysis for $S_{2}$ over the entire program, to discover that the assignment to pLen on edge $4 \rightarrow 6$ may cause some paths that traverse all edges in $S_{2}$ not to preserve the property. This edge thus forms the interference set for sufficient set $S_{2}$, denoted as $I_{2}=\{4 \rightarrow 6\}$ (Cf. Sec. 3.4). Any path program that traverses all the edges in $S_{2}$ but not the interference edge $4 \rightarrow 6$ satisfies the property.

Termination: SMPP has now computed sufficient-interference pairs of sets $\left(S_{1}, \emptyset\right)$ and $\left(S_{2}, I_{2}\right)$, extracted from the two path programs considered. Every path program is covered by these sets, i.e, each program path must either traverse every edge in $S_{1}$, or traverse every edge in $S_{2}$ while traversing no edge in $I_{2}$. Recall that the edges that are part of the loop $10 \leadsto 10$ are part of an MSCC and thus not considered in enumerating path programs. As a result, by examining 2 out of the 4 possible path programs, SMPP has established the property. This computation occurs over Algo. 1 lines 10, 3,11 .

Symbolic Encoding: Fig. 2 consists of a small program with a few path programs. In practice, we have observed CFGs with as few as 500 edges that exhibit millions of path programs. Therefore, reasoning about sets of path progams explicitly is not feasible.

We overcome this difficulty by means of a symbolic encoding of control paths associated with path programs. The analysis uses a succinct propositional (Boolean) encoding that supports the following operations efficiently:

1. Encode all of the control paths between two nodes (in the MSCC graph) as a Boolean formula. Such an encoding is linear in the size of the CFG. Sec. 3.1 details this encoding.

2. Given a set $P$ of unexplored control paths, represented as a Boolean formula $\Lambda_{P}$, and a sufficient-interference pair of edge sets $(S, I)$, subtract all of the paths from $P$ that traverse all the edges in $S$ and none of the edges in $I$. Subtraction is performed by adding blocking clauses to the formula $\Lambda_{P}$. 


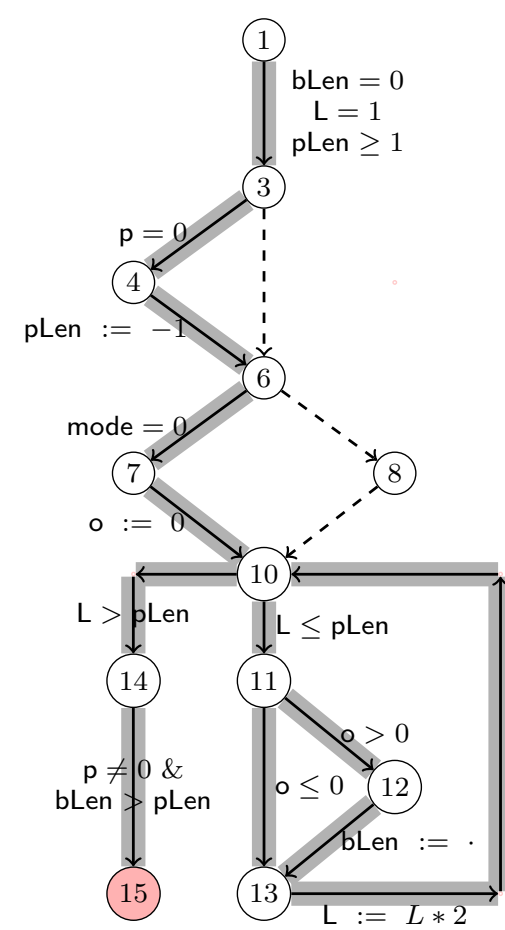

(A)

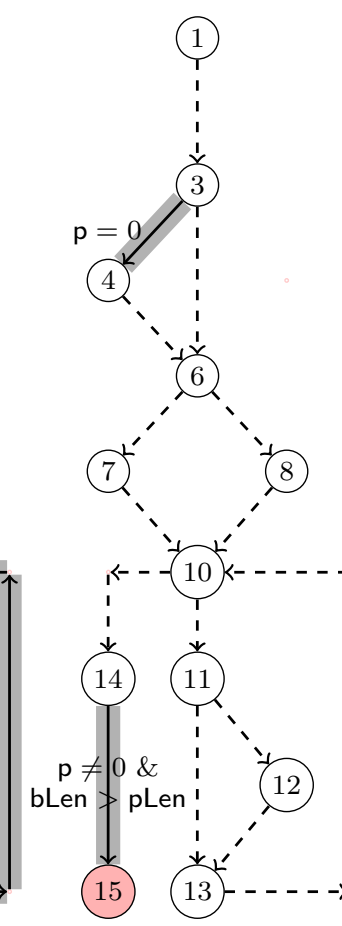

(B)

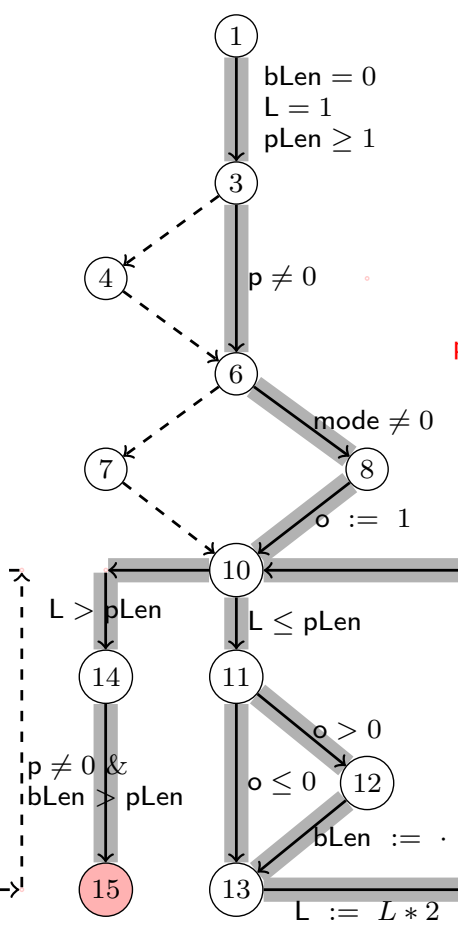

(C)

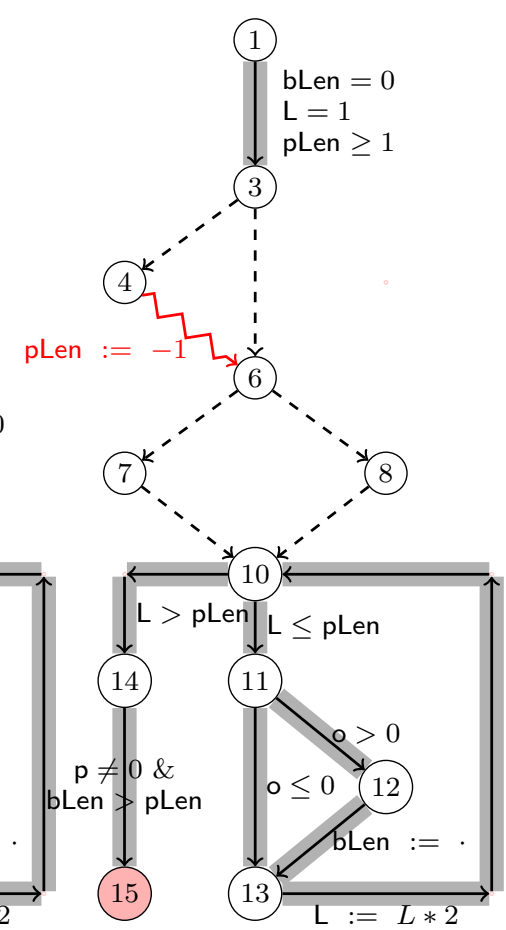

(D)

Figure 2: Path programs enumerated on running example by SMPP. A: Initial path program; B: Sufficient edges for (A); C: Second path program; and D: Sufficient (shaded) and interference (zigzag) edges for (C).

3. Determine that there are no control paths left to examine, or produce an unexplored path. This is equivalent to checking the satisfiability of the updated Boolean SAT formula $\Lambda_{P}$.

SAT solvers have made impressive advances in the past decade, enabling them to support these operations for formulas with hundreds of thousands of variables and clauses. In this respect, our encoding is empirically shown to be quite amenable to existing solvers such as ZChaff [28], even for large programs.

\section{PRELIMINARIES}

We first present our approach on single-procedure programs without function calls. We assume that all variables are of type integer. The handling of function calls (including recursion), non-integer types (especially pointers) and other features present in a programming language such as $\mathrm{C}$ is discussed in Sec. 4. Control-flow graphs (CFG) are used to represent imperative programs.

Control-Flow Graph A CFG $\Pi=\left\langle N, E, V, \rho, n_{0}, n_{f}, \Theta\right\rangle$ is a tuple where $N$ is a set of nodes, $E \subseteq N \times N$ is a set of edges, $n_{0} \in N$ is an initial location, $\bar{n}_{f} \in N$ is a final location, and $V$ is a set of program variables. Each edge $e \in E$ is labeled by a transition relation $\rho_{e}\left(V, V^{\prime}\right)$, a firstorder assertion over current-state variables $V$ and next-state variables denoted by $V^{\prime}$. The first-order assertion $\Theta$ specifies the initial values of the program variables.

An execution of the CFG starts at the initial location with initial values to the program variables that satisfy $\Theta$, and terminates when it reaches the final location. A program assertion $\langle n, \psi\rangle$ for $n \in N$ and assertion $\psi$ over the program variables, requires that $\psi$ hold whenever control reaches the node $n$.

Example 2.1. Fig. 1 shows a CFG of a program over integer-valued and pointer variables (note that for this example, we are only interested in the integer value of the pointer itself). The nodes in the CFG are numbered according to the corresponding labels in the program. We wish to prove the unreachability of node 15 which corresponds to the failure of the assertion in the corresponding line of the program. However, a path-insensitive analysis is unable to prove the property. The join operation at line 5 may lose the relation between $\mathrm{p}$ and $\mathrm{pLen}$.

\subsection{Path Programs}

Given a graph $G$ : $\langle N, E\rangle$, a strongly connected component (SCC) consists of a subset of nodes $C \subseteq N$ such that for each $n_{1}, n_{2} \in C$, there exists a path from $n_{1}$ to $n_{2}$ and vice-versa. A strongly connected component $C$ is maximal(MSCC) iff no strict superset of $C$ is a strongly connected component. The MSCC-decomposition of a graph $G$ is a directed, acyclic graph $G_{D}$ whose nodes $N=\left\{n_{1}^{d}, \ldots, n_{m}^{d}\right\}$ correspond to each of the MSCCs $C_{1}, \ldots, C_{m}$ of $G$ and whose edges connect $n_{i}^{d}$ and $n_{j}^{d}$ if and only if some node in $C_{i}$ connects to some node in $C_{j}$ by an edge in $E$. The MSCC decomposition of a graph can be computed in linear time [9]. The CFG in Fig. 1 contains a non-trivial MSCC consisting of the nodes $\{10,11,12,13\}$. 
Def. 2.1 (Path Program [4]). Let $\Pi_{D}$ be the $M S C C$ decomposition of a CFG $\Pi$. A path program $\pi: n_{0}^{d} \leadsto n^{d}$ is a simple path in $\Pi_{D}$ from the initial node $n_{0}^{d}$ to a node $n^{d}$. The path program $\pi$ naturally corresponds to a subset of nodes and edges in $\Pi$, obtained as the union of the MSCCs traversed by $\pi$ and the edges that connect them.

\subsection{Inductive Invariants}

Let $\Sigma$ denote the universe of concrete program states and $\Gamma$ be a domain of assertions over the program variables ordered by the logical implication $\models$. Each assertion $\varphi \in \Gamma$ represents a set of states $\llbracket \varphi \rrbracket \in 2^{\Sigma}$. An example of an assertion language used in practice is linear arithmetic formulae over the integer variables of a program.

Inductive Map For a set $N$ of nodes in a control-flow graph, a flow-sensitive map $\eta: N \rightarrow \Gamma$ maps each node to a set of program states, represented as a formula. Such a map is inductive iff the following conditions hold:

$$
\begin{aligned}
\text { Initiation }: & \Theta \models \eta\left(n_{0}\right) \\
\text { Consecution }: & \text { foreach edge } e: n_{i} \rightarrow n_{j}, \\
& \eta\left(n_{i}\right) \wedge \rho_{e}\left(V, V^{\prime}\right) \models \eta\left(n_{j}\right)\left[V \mapsto V^{\prime}\right]
\end{aligned}
$$

In order to establish a property $\langle n, \varphi\rangle$ for a given program, we seek an inductive map $\eta$ such that $\eta(n) \models \varphi$.

\subsection{Abstract Interpretation}

Abstract Interpretation is a powerful and general framework for systematically computing inductive maps for a given program. An inductive map is a fixed point of a monotone operator in a suitable abstract domain. An abstract domain is a lattice $\langle\Gamma, \models\rangle$ that usually denotes an assertion language used to represent sets of states. The abstraction function $\alpha: 2^{\Sigma} \mapsto \Gamma$ and concretization function $\gamma: \Gamma \mapsto 2^{\Sigma}$ link elements of the abstract lattice to concrete sets of states. They are assumed to form a Galois Connection [12].

To compute an inductive map for the set $N$ of controlflow nodes, with initial node $n_{0}$, we start from an initial map $\eta^{0}: N \rightarrow \Gamma$ and iterate to produce a sequence of maps $\eta^{1}, \eta^{2}, \ldots$, wherein

$\eta^{0}(n):\left\{\begin{array}{l}\top, n=n_{0}, \\ \perp, \text { else }\end{array} \quad \eta^{i+1}\left(n_{b}\right):\left\{\begin{array}{l}\top, n_{b}=n_{0}, \\ \bigsqcup_{n_{a} \rightarrow n_{b}} \text { post } \begin{array}{l}\left(\eta^{i}\left(n_{a}\right),\right. \\ \left.n_{a} \rightarrow n_{b}\right)\end{array}\end{array}\right.\right.$

The iteration terminates when $\forall n \in N . \eta^{i+1}(n) \models \eta^{i}(n)$. Convergence is guaranteed if the domain $L$ satisfies the ascending chain condition. Failing this, widening and narrowing operators can be used to guarantee convergence. If $\eta$ is the fixed point obtained upon convergence, then $\gamma \circ \eta$ is an inductive invariant map.

Abstract domains such as the interval domain [10], octagon domain [27], symbolic intervals [30] and polyhedra [13] can be used to compute useful invariants over the program variables in order to prove properties of interest. Together, these domains represent various levels of trade-off between the strength of the invariants against the complexity of the analysis.

\section{PATH PROGRAM ENUMERATION}

Let $\Pi=\left\langle N, E, V, \rho, n_{0}, n_{f}, \Theta\right\rangle$ be a control flow graph with MSCC decomposition $\Pi_{D}:\left\langle N_{D}, E_{D}\right\rangle$. Let $\Psi:\langle n, \varphi\rangle$ be a property under verification. We present a procedure for symbolically enumerating all control paths from $n_{0}$ to $n_{f}$, verifying $\Psi$ along each associated path program.

Consider Algorithm 1, which shows the overall verification algorithm. The major steps of the algorithm are (a) encoding the set of (unexplored) control paths as a Boolean formula $\Lambda_{\Pi}$ (line 2), (b) iterating over the solutions of $\Lambda_{\Pi}$ (line 3), (c) analyzing each path program associated with the current solution (line 5), (d) extracting sufficient sets, if correct (line 7), otherwise handling violations (line 8). The rest of this section presents the key steps in detail ${ }^{1}$.

\subsection{SAT Encoding}

Path programs are represented by simple paths in the MSCC graph $\Pi_{D}$ of the CFG $\Pi$. We now present a Boolean SAT-based encoding of all control paths in $\Pi_{D}$. This encoding corresponds to an implementation of SatEncodePaths and SolveAndObtainPathPrograms used in Algo. 1.

For each edge $e$ in $\Pi_{D}$, the propositional variable $p_{e}$ indicates if $e$ belongs to the path program. Let out $(m)$ denote the outgoing edges in $E_{D}$ for a node $m \in N_{D}$ and in $(m)$ denote the incoming edges. Let $\operatorname{src}(e)$ and $\operatorname{tgt}(e)$ denote the source and target nodes of an edge $e \in E_{D}$, respectively. For a set $E_{s} \subseteq E_{D}$, the formula exactlyOne $\left(E_{s}\right)$ denotes that exactly one edge from $E_{s}$ occurs in a path: exactlyOne $\left(E_{s}\right): \bigvee_{e \in E_{s}} p_{e} \wedge \bigwedge_{e, f \in E_{s}, e \neq f} p_{e} \Rightarrow \neg p_{f}$. The formula $\Lambda_{\Pi}$ is the conjunction of all Boolean constraints given in Table 1. The constraints shown in the table enforce that any path program has (a) a visit to the initial node $n_{0}$, (b) a visit to the final node $n_{f}$, (c) exactly one incoming edge to each node $n$ visited (with the exception of $n_{0}$ ), and (d) exactly one outgoing edge from a node $n$ that is visited (with the exception of $n_{f}$ ).

Theorem 3.1. The Boolean formula $\Lambda_{\Pi}$ encodes all paths between $n_{0}$ and $n_{f}$ in the MSCC decomposition $\Pi_{D}$ of $\Pi$.

\subsection{Extracting Sufficient Sets}

We now describe AnalyzePathPrograms and ExtractSufficientSet as used in Algo. 1. Let $\Pi$ be the original CFG, $n_{0}$ be the initial node and $\left\langle n_{f}, \varphi\right\rangle$ be the property we wish to establish. Given a path program $\pi: n_{0}^{D} \leadsto n_{f}^{D}$, SMPP analyzes $\pi$ using a proof-generating oracle. If the property holds over $\pi$, we extract a set of sufficient edges. Otherwise, failure to prove the property indicates a potential violation - details of handling violations are provided in Sec. 3.6. We now present the extraction of sufficient edges from proofs.

Let $\Pi_{\pi}:\left\langle N_{\pi}, E_{\pi}\right\rangle$ represent the subset of the original CFG induced by the path program $\pi$. The proof-generating oracle yields a proof of safety in the form of an inductive invariant map (fixed-point) $\eta: N_{\pi} \mapsto \Gamma$ over $G_{\pi}$. The map $\eta$ maps each node $n \in N_{\pi}$ to an invariant $\eta(n)$, valid over all executions along $\pi$. If $\eta(n) \models \varphi$, then none of the executions leading from $n_{0}$ to $n_{f}$ along the nodes and edges in $\pi$ lead to a violation.

We extract sufficient edges which form the "core reason" behind our proof as follows: (A) we first prune away unnecessary invariants from the map $\eta$ using the notion of a minimal supporting set. Removing such invariants is essential to obtain a compact set of sufficient edges. (B) We then compute an inductive map $\eta^{\prime}$ that supports this set. (C) From $\eta^{\prime}$, we directly extract a sufficient set of edges.

\footnotetext{
${ }^{1}$ Proofs of key results are provided in the extended version of this paper available from the authors upon request.
} 
Table 1: Boolean encoding of the set of all path programs (i.e, paths through the MSCC DAG).

\begin{tabular}{|l|l|}
\hline Fact & Encoding \\
\hline$n_{0}$ should be visited & exactlyOne $\left(\operatorname{out}\left(n_{0}\right)\right)$ \\
$n_{f}$ should be visited & exactlyOne $\left(\operatorname{in}\left(n_{f}\right)\right)$ \\
Source of each edge has exactly one predecessor $\left(\right.$ except for $\left.n_{0}\right)$ & $\bigwedge_{e \in E_{D}, \operatorname{src}(e) \neq n_{0}}\left[p_{e} \Rightarrow \operatorname{exactlyOne}(\operatorname{in}(\operatorname{src}(e)))\right]$ \\
Target of edge has exactly one successor $\left(\right.$ except for $\left.n_{f}\right)$ & $\bigwedge_{e \in E_{D}, \operatorname{tgt}(e) \neq n_{f}}\left[p_{e} \Rightarrow \operatorname{exactlyOne}(\operatorname{out}(\operatorname{tgt}(e)))\right]$ \\
\hline
\end{tabular}

Example 3.1 (Unnecessary Invariants). Consider the "second" path program $\pi_{2}$ shown in Fig. 2(C):

$$
1 \rightarrow 3 \rightarrow 6 \rightarrow 8 \rightarrow\{10,11,12,13\}^{*} \rightarrow 14 \rightarrow 15 .
$$

A polyhedral domain abstract interpreter computes the following invariant:

$$
\eta(14):\left[\begin{array}{l}
\mathrm{p} \neq 0 \wedge \mathrm{pLen} \geq 1 \wedge \\
2 \cdot \mathrm{bLen} \leq \mathrm{L} \wedge \mathrm{bLen} \leq \mathrm{pLen} \\
\text { mode } \neq 0 \wedge \mathrm{o}=1
\end{array}\right] .
$$

This invariant establishes the required unreachability of node 15 for the path program $\pi_{2}$. Nevertheless, the entire invariant is not required for the proof. The sole invariant bLen $\leq$ pLen (underlined above) suffices. The remaining invariants are extraneous.

In principle, we can use other techniques that (attempt to) generate minimal sets of invariants required to prove a given property $[6,18,19]$. These techniques can generate strong invariants for small but complex loops. However, they are currently unsuitable for generating simple global invariants for large path programs. We provide a generic scheme described below utilizing a fixed point, wherein the fixed point can be computed in any way (including predicate abstraction over path programs).

\section{Generalizing Invariants}

To describe how to generalize an invariant map, we first describe the computation of minimal support sets, a key primitive. Let $Q:\left\{q_{1}, \ldots, q_{m}\right\}$ and $q$ be assertions over program variables in a suitable logical theory, such that $q_{1} \wedge q_{2} \wedge \ldots \wedge q_{m} \models q$.

Def. 3.1 (Minimal Support Set). A subset $Q^{\prime} \subseteq Q$ supports the inference $\bigwedge_{q_{i} \in Q} q_{i} \models q$ iff $\bigwedge_{q_{j} \in Q^{\prime}} q_{j} \models q$. A support set $Q^{\prime}$ is minimal iff no proper subset of $Q^{\prime}$ can support the inference.

For $\psi_{1} \models \psi_{2}$, let MinSupport $\left(\psi_{1}, \psi_{2}\right)$ denote the set of minimal supporting conjuncts in $\psi_{1}$ that imply $\psi_{2}$. An implementation of MinSupport (through unsatisfiable cores) is available in existing solvers for many useful theories such as linear arithmetic.

Example 3.2. The assertions $q_{1}: i \geq j, q_{2}: j \geq k+1$, $q_{3}: i \geq k+1, q_{4}: k \geq 1$ together imply the assertion $q$ : $i \geq 2$ in the theory of linear arithmetic over integers. Note that the subset $\left\{q_{1}, q_{2}, q_{4}\right\}$ by itself (and no proper subset thereof) suffices to establish $q$ and is thus a minimal support set. The minimal support set is not unique. The set $\left\{q_{3}, q_{4}\right\}$ also forms a minimal support set.

We assume that the abstract domain $\Gamma$ is a "Moore-closed domain." Specifically, each invariant $\varphi$ is a finite conjunction of a set of atomic predicates that are negation closed: $\varphi$ : $q_{1} \wedge q_{2} \cdots \wedge q_{m}$. Inductive invariants that consist of only conjunctive assertions suffice, in general, to prove any given property. Proofs involving disjunctions of conjunctions can be transformed into purely conjunctive proofs on a suitable elaboration of the original program [31].

Let $\eta$ be a fixed-point map that establishes a property $\left\langle n_{f}, \varphi\right\rangle$, i.e., $\eta\left(n_{f}\right) \models \varphi$. Since $\eta$ is a fixed-point, for every edge $e: n_{1} \rightarrow n_{2} \in E_{\pi}$, the following consecution condition holds:

$$
\eta\left(n_{1}\right) \wedge \rho_{e}\left(V, V^{\prime}\right) \models \eta\left(n_{2}\right)\left[V \mapsto V^{\prime}\right] .
$$

Our overall strategy to generalize $\eta$ is to construct a finite sequence of maps $\mu^{0}, \ldots, \mu^{N}$, wherein the initial map is defined as:

$$
\mu^{0}(m)=\left\{\begin{array}{ll}
\operatorname{MinSupport}\left(\eta\left(n_{f}\right), \varphi\right) & m=n_{f} \\
\text { true } & m \neq n_{f}
\end{array} .\right.
$$

The initial map $\mu^{0}: N_{\pi} \mapsto L$ maps the node $n_{f}$ to the minimal support set that enables $\eta\left(n_{f}\right)$ to prove the required property $\left\langle n_{f}, \varphi\right\rangle$ and maps all other nodes to true. The iterative process $\mu^{0} \ldots, \mu^{N}$ will converge onto a final map $\mu^{N}$ that establishes the property $\left\langle n_{f}, \varphi\right\rangle$, contains no redundant invariants, and generalizes $\eta$.

The intermediate maps $\mu^{i}$ for $i<N$ need not be inductive. For instance, the map $\mu^{0}$ could fail the consecution property for the incoming edges to the node $n_{f}$. The maps $\mu^{i}, i \in$ $[0, N]$ have the following properties:

(a) $\mu^{N}$ is inductive and proves the property $\left\langle n_{f}, \varphi\right\rangle$.

(b) For each $\mu^{i}$, and for each node $m$, the assertion $\mu^{i}(m)$ consists of a subset of conjuncts from $\eta(m)$. As a result $\eta(m) \models \mu^{i}(m)$.

(c) Each successive map incorporates at least as many conjuncts from $\eta$ as the previous, i.e, $\forall i<j, m \in N_{\pi} \cdot \mu^{j}(m) \models$ $\mu^{i}(m)$.

We propose a process called local repair to derive the map $\mu^{i+1}$ from $\mu^{i}$.

Local Repair: We address the failure of $\mu^{i}$ for $i<N$ to be an inductive invariant by means of local repair. To perform the local repair of $\mu^{i}$, we strengthen $\mu^{i}(a)$ for some node $a$ to address the failure of consecution along an edge $e: a \rightarrow b$ : $\mu^{i}(a) \wedge \rho_{a \rightarrow b}\left(V, V^{\prime}\right) \not \mu^{i}(b)$. However, $\eta(a) \wedge \rho_{e}\left(V, V^{\prime}\right) \models$ $\mu^{i}(b)^{2}$. Let $Q_{a}$ be a minimal subset of conjuncts from $\eta(a)$ that supports this implication. The local repair of $\mu^{i}(a)$ w.r.t $a \rightarrow b$ is $\mu^{i+1}(a)=\operatorname{MinSupport}\left(\eta(a)\right.$, pre $\left(\mu^{i}(b), e: a \rightarrow\right.$ $b)$. The application of MinSupport removes redundant conjuncts from the assertion $\eta^{i}(a)$. Thus $\mu^{i+1}(a)$ minimally supports consecution across the edge $a \rightarrow b$. Strengthening $\because \eta(a) \wedge \rho_{e}\left(V, V^{\prime}\right) \models \eta(b) \models \mu^{i}(b)$ 
$\mu^{i}(a)$ for some node $a$ may invalidate the consecution condition for some of its incoming edges. A new repair iteration is then required to address this failure. This process converges when $\mu^{i}$ is inductive, thus needing no further iteration.

Theorem 3.2. The process of repeated local repair terminates in finitely many steps yielding a fixed-point map $\mu$, s.t. $\eta(b) \models \mu(b)$ for all $b \in N_{\pi}$.

Example 3.3. Consider again the path $\pi$ from Ex.3.1. Abstract interpretation computes an invariant relating program variables, including $\mathrm{pLen,} \mathrm{bLen,} \mathrm{p}$, and $\mathrm{L}$. The result of the repair iteration for the invariant bLen $\leq$ pLen at node 14 leads to the map $\mu$ partially depicted as:

\begin{tabular}{|r|c|c|c|c|}
\hline$n$ & 3 & 10 & 11 & $13 \& 14$ \\
\hline$\mu(n)$ & $\begin{array}{c}\mathrm{bLen}=0 \\
\mathrm{pLen} \geq 1 \\
\mathrm{~L}=1\end{array}$ & $\mathrm{bLen} \leq \mathrm{pLen}$ & $\begin{array}{c}\mathrm{L} \leq \mathrm{pLen} \\
\mathrm{bLen} \leq \mathrm{pLen}\end{array}$ & $\mathrm{bLen} \leq \mathrm{pLen}$ \\
\hline
\end{tabular}

The result $\mu$ of the repair iteration is used to extract a set of sufficient edges, $S_{\pi} \subseteq E_{\pi}$ that are sufficient for the proof of $\Psi$.

Def. 3.2 (Sufficient Edges). An assignment $e: a \stackrel{x:=\mathrm{e}}{\longrightarrow}$ $b$ is a supporting edge w.r.t $\mu$ if $\mu(b)$ contains an invariant assertion involving the variable $x^{3}$. A condition $e: a \stackrel{q(\mathrm{e})}{\longrightarrow} b$ is a supporting edge if $\mu(a) \not \models q(\mathrm{e})$ and $\mu(b) \models q(\mathrm{e})$.

A sufficient set for $\mu$ is the set of all such edges. The definition of sufficient edges immediately implies a method of deriving such a set of edges from a map $\mu$.

Example 3.4. Continuing Ex.3.3, the sufficient edges corresponding to the proof over $\pi$ consist of the assignments $1 \rightarrow 3$ and conditions $10 \rightarrow 14$ and $14 \rightarrow 15$.

\subsection{Over-Approximate Symbolic Execution}

In many cases, the path program may not contain loops, or the loops present do not affect the property of interest. In such cases, we propose to use symbolic execution along the path program as an oracle. The power of symbolic execution lies in the ability of fast SMT solvers to reason about the feasibility of large formulae in theories such as linear arithmetic or bit-vectors, and in case of infeasibility to quickly extract minimal unsatisfiable cores. Note that standard symbolic execution in general cannot reason about all paths through a program loop. Therefore, we use an over-approximate symbolic execution (described below). If it succeeds in proving the property, we directly obtain a sufficient set. If it fails, then we resort to a more general proof technique like abstract interpretation.

An over-approximate symbolic execution of the path program $\pi$ constructs a formula $\psi_{\pi}$ in a suitable logical theory. This formula is derived by composing the transition relations along the edges in the path program $\pi$. Assignments belonging to loops are treated as assigning a non-deterministic value, and conditions present in loops are treated as nondeterministic choices. Finally, for the property $\langle n, \varphi\rangle$, we assert $\neg \varphi$ as a condition encountered at the node $n$.

Theorem 3.3. If the over-approximate symbolic execution of a path program $\pi$ yields an unsatisfiable formula $\psi_{\pi}$, then any execution of the path program $\pi$ satisfies $\langle n, \varphi\rangle$.

\footnotetext{
${ }^{3}$ Alternatively, the consecution post $(\mu(a), e) \models \mu(b)$ should cease to hold if the assignment is made non-deterministic.
}

Example 3.5. Symbolic execution of the "first" path program $\pi_{1}: 1 \rightarrow 3 \rightarrow 4 \rightarrow 6 \rightarrow 7 \rightarrow\{10,11,12,13\}^{*} \rightarrow$ $14 \rightarrow 15$, shown in Fig. 2(A) (originally from Ex.2.1) yields the following formula obtained by composing the transition relations of the individual edges:

$$
\left[\begin{array}{l}
\rho_{1,3}:\left(\text { bLen }_{0}=0 \wedge \mathrm{pLen}_{1} \geq 1 \wedge \mathrm{pLen}_{1} \geq \mathrm{pLen}_{0}\right) \wedge \\
\rho_{3,4}:\left(\mathrm{p}_{0}=0\right) \wedge \rho_{4,6}:\left(\mathrm{pLen}_{1}=-1\right) \wedge \\
\rho_{6,7}:\left(\text { mode }_{0}=0\right) \wedge \rho_{7,10}:\left(\mathrm{i}_{0}=0\right) \wedge \\
\rho_{10,14}:(\mathrm{L}>\mathrm{pLen} \\
\left.\rho_{14,15}\right) \\
\rho_{14}\left(\underline{\mathbf{p}_{0} \neq 0} \wedge \text { bLen }_{1}>\mathrm{pLen}_{1}\right)
\end{array}\right]
$$

The subscripts on the variables occurring in the transitions are derived from an SSA-form of the program $\xi$ or using a use-def chain analysis. Note that $\psi_{\xi}$ is infeasible, proving that the path $\xi$ satisfies $\Psi$.

Let $\psi: \rho_{1} \wedge \cdots \wedge \rho_{m}$ be an infeasible formula obtained from a path program $\pi$. Furthermore, let $R=\left\{\rho_{i_{1}}, \ldots, \rho_{i_{k}}\right\}$ be an unsatisfiable core for the formula $\psi$ and $S=\left\{e_{i_{1}}, \ldots, e_{i_{k}}\right\}$ be the subset of edges that yield the transitions in the set $R$. The set $S$ forms a sufficient set for the infeasibility of $\pi$.

Example 3.6. Returning to Ex.3.5, the unsatisfiable core consists of the transition relation $\rho_{3,4}$ along with $\rho_{14,15}$. This yields the sufficient set $S=\{3 \rightarrow 4,14 \rightarrow 15\}$.

\subsection{Interference Analysis}

Thus far, we have focused on the analysis of a single path program. Interference analysis extends the learning due to sufficient edges extracted from a given path program, to consider other path programs. Thus, interference analysis operates on the entire CFG.

For a path program $\pi$, the sufficient set $S_{\pi} \subseteq E_{\pi}$ represents edges relevant to the proof of the property along $\pi$. Using $S_{\pi}$, we seek to characterize the set of path programs in the original $C F G$ that are also guaranteed to satisfy the property $\Psi$ and are proven by the same sufficient set. To do so, we also need to reason about potentially interfering assignments in the CFG. This corresponds to the component of ExtractSupportSet from Algo. 1 that yields $I_{\pi}$.

Example 3.7. In Ex.3.4, the process of local repair over the path program $\pi_{2}$ in Fig. 2(C) yields a sufficient set $S_{2}$ : $\{1 \rightarrow 3,10 \rightarrow 14,14 \rightarrow 15\}$. The path $\pi_{1}$ shown in Fig. 2(A) traverses all of the edges in the set $S_{2}$, yet the proof of the property obtained along the sufficient set $S_{2}$ does not apply for this path. The reason is that the assignment $\mathrm{pLen}:=-1$ along the edge $4 \rightarrow 6$ invalidates the value of the variable pLen that is initially defined in $1 \rightarrow 3$. Therefore the proof in Ex.3.4 does not apply to this path.

This assignment disrupts the critical use-def chain between edges $1 \rightarrow 3$ where the variable pLen is defined and $10 \rightarrow 11$, where it is used. Our approach is to identify a set of interference edges that can invalidate the use-def chains in the sufficient set.

Def. 3.3 (Interfering Edge). An assignment edge $e \notin$ $E_{\pi}$ assigning variable $x$ is an interference edge for a sufficient set $S_{\pi} \subseteq E_{\pi}$ iff there exist edges $e_{1}, e_{2} \in S_{\pi}$ wherein $e_{1}$ defines $x$, $e_{2}$ uses $x$, and a path of the form $e_{1} \leadsto e \sim e_{2}$ exists in the original CFG.

Returning to Ex.3.7, we verify that the edge $4 \rightarrow 6$ interferes with the def-use chain $e_{1}: 1 \rightarrow 3$ and the condition 
$e_{2}: 10 \rightarrow 11$. Given a path program $\pi$ and the sufficient edges $S_{\pi}$, a set of interfering edges $I_{\pi}$ can be computed using a use-def chain computation and a control reachability analysis on the original CFG. In principle, a finer semantic criterion for interference can be formulated that checks whether an interfering edge preserves the invariants related to the sufficient set. However, in our implementation, the syntactic criterion presented here is used due to its simplicity.

Theorem 3.4. If a property $\Psi:\langle n, \varphi\rangle$ holds on a path program $\pi$ then it holds on any path program $\xi: n_{0} \leadsto n$ visiting all the edges in $S_{\pi}$ and none of the edges in $I_{\pi}$.

\subsection{Blocking Clauses}

We apply the oracles described in Secs. 3.3 and 3.2, in sequence, to a path program $\pi$ in an attempt to obtain a sufficient set $S_{\pi}$. If the oracle obtains a proof, then the interference analysis described in Sec. 3.4 yields a sufficientinterference pair $\left(S_{\pi}, I_{\pi}\right)$ consisting of the sufficient edges and their corresponding interference edges. We now describe BlockingClause from Algo. 1 that applies these sets to rule out other paths with the same proofs of correctness.

The formula BlockPaths $\left(S_{\pi}, I_{\pi}\right): \bigvee_{e \in S_{\pi}} \neg p_{e} \vee \bigvee_{f \in I_{\pi}} p_{f}$, encodes paths that either (a) do not visit every node of $S_{\pi}$, or (b) visit some node of $I_{\pi}$. Adding this formula as a blocking clause to $\Lambda_{\Pi}$ avoids revisiting the same set of sufficient edges. This provides proof-based learning to avoid the enumeration of related path programs.

\subsection{Handling Violations}

We now describe how to handle violations as depicted in Algo. 1 lines $8-9$. One could halt the enumeration upon encountering a violation, but it may be desirable to continue the search for errors that stem from a different cause. To this end, SMPP obtains a path slice for a violation along the lines of Jhala and Majumdar [24]. As a result of path slicing, the analysis obtains a set $S_{\pi} \subseteq E_{\pi}$ of edges that cause the error a set $I_{\pi}$ of edges that may interfere with the use-def chains in $S_{\pi}$. Our treatment of the pair $\left(S_{\pi}, I_{\pi}\right)$ obtained from a potential violation is identical to that obtained from a proof through repair iteration.

Theorem 3.5 (Jhala and Majumdar [24]). Let $S_{\pi}, I_{\pi}$ be as computed by the path slicing technique. For any path $\xi$ that visits all the nodes in $S_{\pi}$ and none of the nodes in $I_{\pi}$, the path $\xi$ violates the property $\langle n, \varphi\rangle$ iff $\pi$ does.

\subsection{Expanding Loops}

A path program, corresponding to a control path in the MSCC decomposition, treats loops monolithically. A given loop is entirely part of a path program wherein arbitrarily many iterations are considered, or alternatively no iterations are considered. In the case when the entire program consists of a single while loop (as is the case with control programs), our technique is equivalent to running the oracle over the entire program. While this can lead to imprecision, this problem can be addressed by unwinding and unrolling a given loop, so that our techniques can reason about specific paths in the loop. Furthermore, our choice of an MSCC decomposition was intended to create an acyclic graph related to the CFG. Our approach can be generalized to use other schemes for creating an acyclic graph such that the paths in this graph are related to fragments in the original CFG.

\section{IMPLEMENTATION}

We have implemented our approach as a part of the FSoft program verification platform for C programs [21, 22]. F-Soft checks C programs for buffer overflows, string API usage, NULL pointer dereferences, user-defined type-state properties, memory leaks and so on. For a detailed description of our modeling of structures, pointers and arrays, see $[22]$.

\section{F-Soft Framework}

The F-Soft front-end flattens structure and union types into simple types, constructs a memory model by providing magic number addresses to storage locations, and instruments the program for properties being checked. Pointer aliasing and arithmetic are handled by modeling their effects over integer variables based on a flow-insensitive points-to analysis.

Example 4.1. Fig. 3 illustrates the construction of a memory model in F-Soft based on the results of a points-to analysis. Our model replaces each local variable $p$ in a function $f$ by a variable $f: p$ with global scope. If $f$ can be called in a recursive context then such a variable is treated as a summary variable.

Corresponding to each pointer variable $p$, we introduce an instrumentation variable star $(p)$ to track the contents of its store. The value of star $(p)$ is non-deterministic if $p$ does not point to a valid location. The expression $* p$ is replaced by its representative star $(p)$. An assignment to the $\mathrm{L}$-value $* p$ is rewritten into an assignment to all the variables $x$ that $p$ can potentially point to. Such an assignment to $x$ is guarded by a condition $p==\& x$ that enforces the points-to relationship. Further details and rationale are provided elsewhere [22, 21].

The initial CFG of the program is simplified considerably through program slicing and constant folding. We then perform a series of flow and context sensitive analyses such as constant folding, interval analysis, and various numerical domain analyses. F-Soft implements many abstract domains in a partially path-sensitive abstract interpretation framework [30, 1]. The analysis uses these domains in combination or in succession to attempt to prove a property, each run of the analysis reusing the invariants obtained by the previous runs. F-soft re-slices the program model based on the properties proved by static analysis, reducing it further. At the end of static analysis, the final CFG with its unproven properties is provided as an input to the SMPP implementation.

\section{SMPP Implementation}

Our implementation follows the description in Algorithm 1 with modifications to accommodate function calls.

The overall goal of treating function calls and returns is to ensure that (a) the enumerated path programs properly match calls and returns; and (b) different paths inside a function can be traversed by a path program upon multiple visits under different calling contexts. This is achieved by first performing a context numbering of the function calls in the CFG. The context numbering scheme described by Whaley and Lam can be used for such a purpose [32]. Each CFG edge $e$ then yields multiple propositions $p(e, c)$ based on the different calling contexts $c$ that the edge may be visited in. The rest of the encoding remains unchanged with 

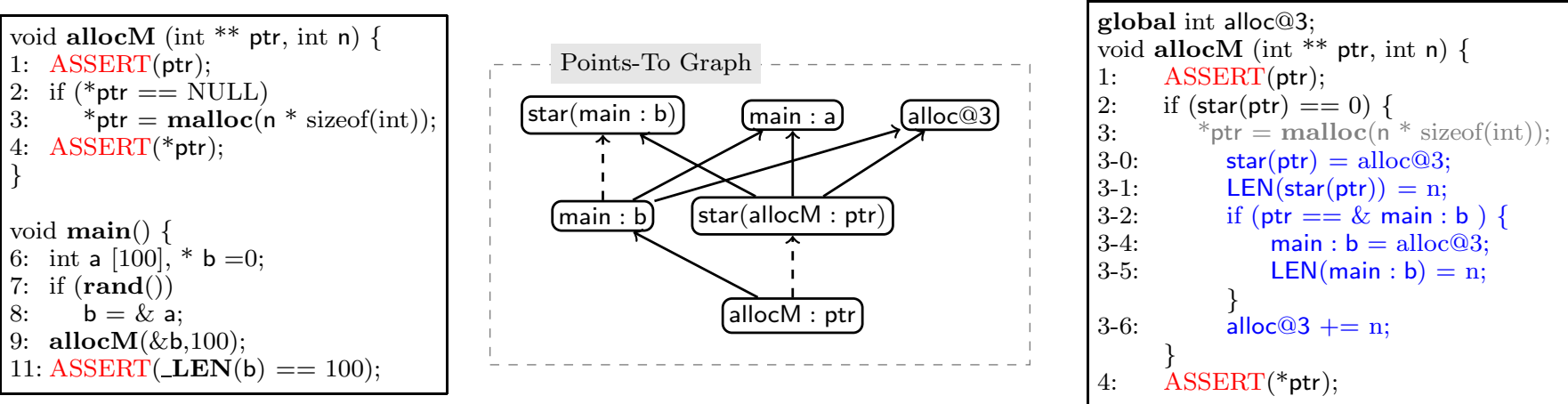

Figure 3: A simplified illustration of F-Soft's memory modeling for a program with pointers.

Table 2: Legend for abbreviations used in Table 3.

\begin{tabular}{ll}
\hline Abbrv. & Remark \\
\hline Blk & Number of blocks at start of SMPP. \\
Prp & Number of properties (asserts). \\
Prf & Number of Proofs. \\
PP tot & Number of path programs total (estimated). \\
PP enum & Number of path programs enumerated by SMPP. \\
PP Proofs & Number of path programs proved correct. \\
SE & Symbolic Execution \\
AI & Abstract Interpretation \\
RI & Repair Iteration
\end{tabular}

the exception of function calls and returns, which now match the calling block context with a return to the appropriate call site.

Recursive calls are currently unwound up to a specified depth and then replaced by a call to a function that returns a non-deterministic value and has non-deterministic sideeffects on variables passed by reference. In practice, this seems to have a minimal impact on the checking of runtime errors.

The enumerated path programs are first analyzed using over-approximate symbolic execution and then (if needed) by the abstract interpreter and a local repair iteration. Currently, the support set and unsatisfiable-core computation are performed using the SMT solver Yices [16]. A failure to prove a property is currently reported as a potential violation. In the future, we plan to integrate our technique with a model checker over path programs to concretize these potential violations.

\section{EXPERIMENTAL EVALUATION}

We conducted experiments to address the following: (a) how efficiently SMPP can verify common safety properties that require path-sensitive reasoning, (b) the effectiveness of proof-based learning for proving many program-paths safe with the analysis of a few, and (c) the power and efficiency of SMPP against another path-sensitive analysis, predicate abstraction.

Fig. 4 presents our experimental setup using the F-Soft framework. The experiments consist of processing the given C program through our front-end which includes path-insensitive, flow- and context-sensitive abstract interpretation through numerical domains such as constants, intervals, and octagons.
Properties proved using these analyses are removed from the CFG, followed by re-slicing and simplification. Thus all properties on which SMPP is tested require some degree of path-sensitive reasoning to validate.

Table 3 presents our experimental results on the Zitser et al. benchmarks [34]. These programs consist of important buffer overflow bugs found in commonly used programs such as wu-ftpd, bind, nslookup and so on, along with the fixes made to them. Note, however, that F-Soft automatically instruments many more properties, as compared to the single line of code that is marked in these benchmarks. The abbreviations used in Table 3 are expanded in Table 2. Table 3 reports the size of each program at the start of SMPP, number of properties, the number of proofs (per oracle), and the time taken (total, percentage for stages of the analysis). Each property that is not a proof is reported as a potential witness along with a sliced path program. For some these potential witnesses, the symbolic execution reported a path program slice that did not include a loop, indicating a concrete witness. The results demonstrate that SMPP can prove a significant number of path-sensitive properties and identify potential witnesses efficiently. Note that symbolic execution is successful in proving a majority of the paths efficiently, and that abstract interpretation is key for a significant portion of properties that involve loops.

In Table 3, the Col. Path Programs (Tot) presents an estimate of the total number of path programs, estimated using SAT (in many cases, the estimator timed out after an hour) while Path Programs (Enum) presents the number actually enumerated by SMPP. The difference demonstrates the effectiveness of proof-based learning.

Table 4 compares the performance of SMPP against Blast v2.5 [3]. To focus on the analyses (rather than on differences in program modeling), we printed the CFG on which $\mathrm{SMPP}$ is applied as a $\mathrm{C}$ program, using goto statements to enforce control flow. The variables in this program are all integers (generated after the F-Soft front-end process of instrumentation, memory modeling, simplifications, slicing and static property proofs). Blast had to be invoked multiple times on the same program, each instance targeting a different property. This was needed to avoid the default aggregation of properties in Blast which failed with errors in many instances. For fair comparison, we ran SMPP multiple times with each instance targeting a single property, recomputing the SAT encodings and the sufficient sets from scratch. The comparison in Table 4 shows that while Blast 
Table 3: Experimental Evaluation on the Zitser et al. Benchmarks on CFG obtained after initial static analysis. The path counter timeout was set to $1 \mathrm{hr}$. Table 2 explains the abbreviations used here.

\begin{tabular}{|c|c|c|c|c|c|c|c|c|c|c|c|c|c|}
\hline \multirow[t]{2}{*}{ Name } & \multirow{2}{*}{$\begin{array}{l}\text { LOC } \\
\text { Orig. }\end{array}$} & \multirow{2}{*}{$\begin{array}{c}\text { Blk } \\
\text { (tot) }\end{array}$} & \multirow{2}{*}{$\begin{array}{l}\text { Prp } \\
\text { (tot) }\end{array}$} & \multirow[t]{2}{*}{$\overline{\operatorname{Prf}}$} & \multicolumn{2}{|c|}{ Path-Programs } & \multicolumn{3}{|c|}{ PP Proofs } & \multicolumn{4}{|c|}{ Times (seconds) } \\
\hline & & & & & Total & Enum & Tot & $\begin{array}{c}\mathrm{SE} \\
\%\end{array}$ & $\begin{array}{c}\mathrm{AI} \\
\%\end{array}$ & $\begin{array}{l}\text { Tot } \\
\text { sec }\end{array}$ & $\begin{array}{l}\mathrm{SE} \\
\%\end{array}$ & $\begin{array}{l}\text { AI } \\
\%\end{array}$ & $\begin{array}{l}\mathrm{RI} \\
\%\end{array}$ \\
\hline \multicolumn{14}{|c|}{ SENDMAIL } \\
\hline s2-ok & 1151 & 744 & 60 & 20 & $>1.2 \mathrm{M}$ & 204 & 131 & 80 & 20 & 136.76 & 7 & 65 & 21 \\
\hline s2-bad & 1132 & 721 & 58 & 21 & $>1.2 \mathrm{M}$ & 259 & 195 & 86 & 14 & 139.17 & 10 & 77 & 3 \\
\hline s4-ok & 776 & 263 & 12 & 6 & 12 & 12 & 6 & 0 & 100 & 10.98 & 2 & 95 & 1 \\
\hline s4-bad & 713 & 284 & 30 & 15 & 30 & 30 & 15 & 0 & 100 & 30.29 & 1 & 96 & 0 \\
\hline s5-ok & 837 & 179 & 14 & 13 & 14 & 2 & 1 & 100 & 0 & 0.5 & 40 & 0 & 0 \\
\hline s5-bad & 810 & 182 & 17 & 16 & 17 & 2 & 1 & 100 & 0 & 0.5 & 40 & 0 & 0 \\
\hline s6-ok & 317 & 121 & 8 & 3 & 8 & 8 & 3 & 0 & 100 & 13.18 & 0 & 98 & 0 \\
\hline s6-bad & 315 & 121 & 8 & 3 & 8 & 8 & 3 & 0 & 100 & 14.91 & 0 & 98 & 0 \\
\hline s7-ok & 1824 & 1357 & 174 & 59 & $>.5 \mathrm{M}$ & 415 & 224 & 92 & 8 & 132.4 & 25 & 48 & 0 \\
\hline s7-bad & 1816 & 1347 & 170 & 94 & $>.6 \mathrm{M}$ & 412 & 271 & 92 & 8 & 135 & 24 & 53 & 0 \\
\hline \multicolumn{14}{|l|}{ BIND } \\
\hline b1-ok & 2177 & 670 & 55 & 10 & $>.4 \mathrm{M}$ & 214 & 168 & $\overline{96}$ & 4 & 179.6 & 9 & 85 & $\overline{0}$ \\
\hline b1-bad & 2117 & 662 & 54 & 9 & $>.4 \mathrm{M}$ & 181 & 135 & 95 & 5 & 171.79 & 9 & 86 & 0 \\
\hline b2-ok & 2706 & 957 & 80 & 11 & $>.4 \mathrm{M}$ & 285 & 216 & 97 & 3 & 282.23 & 23 & 69 & 0 \\
\hline b2-bad & 2688 & 955 & 80 & 11 & $>.4 \mathrm{M}$ & 289 & 220 & 97 & 3 & 230.14 & 33 & 59 & 0 \\
\hline \multicolumn{14}{|c|}{ WU-FTPD } \\
\hline f1-ok & 628 & 144 & 13 & 4 & 188 & 28 & 14 & 71 & 29 & 11.81 & 2 & 88 & 8 \\
\hline f1-bad & 562 & 178 & 21 & 9 & 481 & 71 & 52 & 92 & 8 & 25.14 & 3 & 91 & 4 \\
\hline f2-ok & 1208 & 119 & 9 & 5 & 5637 & 17 & 13 & 100 & 0 & 0.25 & 68 & 20 & 0 \\
\hline f2-bad & 936 & 114 & 8 & 4 & 2175 & 14 & 10 & 100 & 0 & 0.19 & 72 & 16 & 0 \\
\hline
\end{tabular}
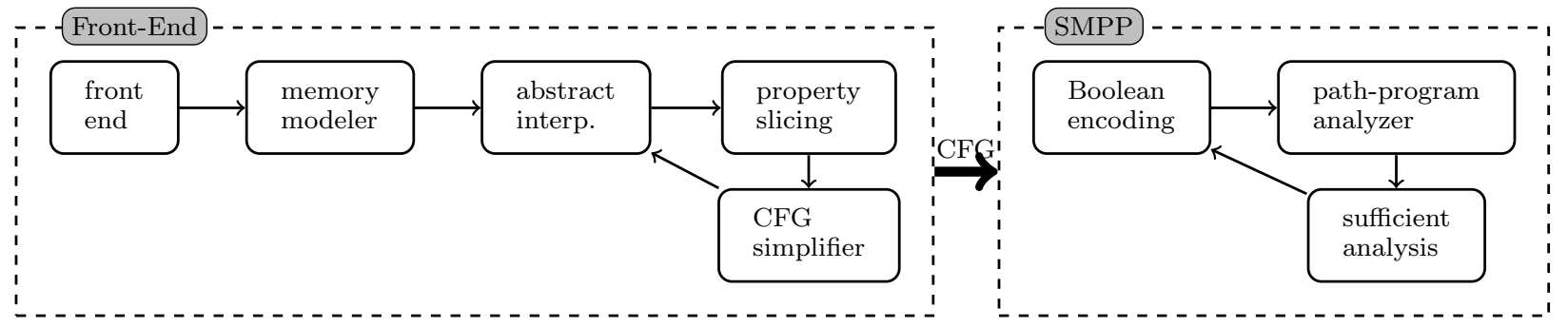

Figure 4: Experimental setup: Front-end includes path-insensitive analyses, followed by SMPP.

can prove more properties than SMPP in some cases, our coarser control-based abstraction can be much faster and finds more violations in many cases. A majority of the failures by Blast resulted from an explosion in the number of predicates on loops (termed "Gremlins"). Our abstract interpretation followed by a proof-based learning seems to be adequate for many such cases.

Analysis of Larger Benchmarks. Table 5 summarizes our experimental results on larger case studies. The experimental setup remains the same as in Fig. 4. Program sources were downloaded from the internet and first analyzed using our tool SpecTackle [22], which infers likely preconditions and post-conditions corresponding to pointer and array accesses in the functions ${ }^{4}$. F-Soft (with SMPP) is then invoked for each function in the program. The preconditions for the entry functions are assumed, whereas the preconditions for called functions are asserted. In order to control the CFG size, calls to functions not reachable from the entry function within a context of depth 4 were replaced by non-deterministic choice. Table 5 shows the results. The

\footnotetext{
${ }^{4}$ These benchmarks along with the inferred preconditions are available upon request.
}

SMPP approach is invoked only in those cases where there are unresolved properties from the initial path-insensitive analyses. This accounts for roughly $40 \%$ of the functions, on average. The total time taken by the SMPP approach is of the same order as that taken by the initial model construction and static analysis phases. In almost all cases, the overall analysis (initial+SMPP) terminates within the given time limit of 45 minutes. Note that SMPP detects a significant number of extra proofs, over and above sophisticated flow- and context-sensitive polyhedral abstract interpretation techniques. We did not run Blast on these larger case studies, as it would have required significant manual effort in instrumenting the CFGs generated by our front-end.

\section{RELATED WORK}

Abstraction Refinement . Our technique fits broadly into the abstraction refinement paradigm. The Boolean formulae representing all unexplored control paths is a coarse controlflow abstraction. This abstraction is successively refined by eliminating the path programs proved correct.

The main differences from typical abstraction refinement approaches are: (a) Our abstraction is based solely on con- 
Table 5: Results on Larger Open-Source Case-Studies

\begin{tabular}{|c|c|c|c|c|c|c|c|c|c|c|c|c|c|}
\hline \multirow[t]{3}{*}{ Name } & \multirow[t]{3}{*}{ KLOC } & \multirow[t]{3}{*}{ \#Fun } & \multirow[t]{3}{*}{ \#Prec } & \multicolumn{4}{|c|}{ Front-End } & \multicolumn{6}{|c|}{ SMPP } \\
\hline & & & & Blk & Time & Prp & $\overline{\text { Prf }}$ & & Fun & $\overline{B l k}$ & $\# \mathrm{PP}$ & Prf. & Time \\
\hline & & & & avg & $\mathrm{sec}$ & tot & tot & tot & timeout & avg & tot & tot & tot, sec \\
\hline thttpd-2.25b & 14.7 & 172 & 1901 & 263 & 5162 & 12161 & 11069 & 68 & 1 & 199 & 1622 & 512 & 1393 \\
\hline ssh-server-4.1 & 30.1 & 313 & 2047 & 190 & 7755 & 42773 & 41182 & 127 & 5 & 120 & 1681 & 564 & 1197 \\
\hline xvidcore & 63.9 & 350 & 6127 & 520 & 13259 & 17219 & 12020 & 190 & 6 & 331 & 5220 & 2090 & 15728 \\
\hline
\end{tabular}

Table 4: Comparison of SMPP with BLAST. Prp: number of properties (asserts), Wit: number of concrete witnesses, and Fail: Blast failures. Note that SMPP is run multiple times (once for each property) for fairer comparison.

\begin{tabular}{||l|r||r|l||r|r|r|r||}
\hline \multirow{2}{*}{ Name } & \multicolumn{1}{|c||}{ Prp } & \multicolumn{2}{c||}{ SMPP } & \multicolumn{4}{c||}{ BLAST } \\
\cline { 3 - 7 } & & Prf & Time & Prf & Wit & Fail & Time \\
\hline s2-ok & 60 & 20 & $2 \mathrm{~m} 10 \mathrm{~s}$ & 35 & 0 & 25 & $2 \mathrm{~h} 7 \mathrm{~m}$ \\
s2-bad & 58 & 21 & $2 \mathrm{~m} 25 \mathrm{~s}$ & 35 & 0 & 23 & $1 \mathrm{~h} 50 \mathrm{~m}$ \\
s4-ok & 12 & 6 & $0 \mathrm{~m} 11 \mathrm{~s}$ & 11 & 1 & 0 & $0 \mathrm{~h} 1.3 \mathrm{~m}$ \\
s4-bad & 30 & 15 & $0 \mathrm{~m} 31 \mathrm{~s}$ & 17 & 1 & 12 & $2 \mathrm{~h} 8 \mathrm{~m}$ \\
s5-ok & 14 & 13 & 0m00.5s & 6 & 0 & 8 & $0 \mathrm{~h} 55 \mathrm{~m}$ \\
s5-bad & 17 & 16 & 0m00.5s & 9 & 0 & 8 & $1 \mathrm{~h} 32 \mathrm{~m}$ \\
s6-ok & 8 & 3 & $0 \mathrm{~m} 14 \mathrm{~s}$ & 4 & 0 & 4 & $0 \mathrm{~h} 17 \mathrm{~m}$ \\
s6-bad & 8 & 3 & $0 \mathrm{~m} 15 \mathrm{~s}$ & 4 & 0 & 4 & $0 \mathrm{~h} 17 \mathrm{~m}$ \\
s7-ok & 174 & 59 & $28 \mathrm{~m}$ & 135 & 1 & 38 & $17 \mathrm{~h} 22 \mathrm{~m}$ \\
s7-bad & 170 & 94 & $27 \mathrm{~m}$ & 134 & 1 & 35 & $15 \mathrm{~h} 55 \mathrm{~m}$ \\
\hline
\end{tabular}

trol locations. This is a very inexpensive abstraction to compute, deferring the heavier work to an oracle for checking correctness of a path program corresponding to the enumerated control path. Other approaches typically use an explicit representation of the control flow with data predicates, e.g. Boolean programs $[2,3]$. Such abstractions are more expensive to compute than our abstractions. (b) Rather than a refinement loop over false error traces (counterexamples) [8, 2 ], our refinement loop operates over path programs associated with the enumerated control paths. (c) Our approach avoids divergences on loops in the program. This is because we enumerate over an acyclic MSCC-based graph derived from the CFG, wherein each control path corresponds to a path program that can potentially capture infinitely many control paths on the original CFG. It is well known that effective handling of loops is a stumbling block for many abstraction refinement techniques. Abstract interpretation techniques using widening have been well-optimized to prove common types of run-time properties, even in the presence of loops. (d) The decomposition of the overall program into multiple path programs allows flexibility in choosing a suitable oracle for individual sub-problems. In our implementation, we use symbolic execution to handle path programs without loops, and abstract interpretation to handle path programs with loops. Other oracles, such as predicate abstraction refinement, can also be used.

Our work is closely related to recent work by Heizmann et al [20] . They propose an abstraction refinement scheme for trace abstractions. An over-approximation of the set of possible traces is successively refined by means of an interpolant automaton that recognizes a set of infeasible traces. The interpolant automata are also derived from proof techniques that can generate Floyd-Hoare style inductive invariants. The main differences include: (a) our technique operates on path programs as opposed to error traces; (b) we employ a symbolic encoding using SAT to keep track of unexplored path programs, as opposed to an explicit representation.

Lazy abstraction with interpolants by McMillan [26] provides a lazy scheme to refine an abstract model on demand, by utilizing interpolants derived from refuting paths in the program. This work also avoids the cost of an expensive abstraction. However, the refinement is driven by error traces, the control flow is handled explicitly, and details of proofbased learning are different from our technique.

The notions of path programs in our work are directly inspired by the work of Beyer et al. [4]. That work also employs path programs and invariants to avoid loop divergences. In contrast, (a) we use Boolean formulae abstracting just the control flow, as opposed to predicate abstraction (Boolean programs), and (b) we refine through blocking clauses rather than using invariants as predicates. Using blocking clauses for refinement is potentially more scalable than using invariants as predicates. First, invariant generation techniques typically generate a large set of invariants, a majority of which are redundant. Beyer et al. [4] do not attempt to minimize the set of invariants. Our work provides this reduction by means of the local repair iteration discussed in Section 3. Second, each predicate added can potentially double the complexity of model checking an abstraction, whereas our conflict clauses are small and seem to have very little impact on the size of the Boolean formula abstraction. Another approach is to use abstract interpretation to derive useful program invariants as a pre-processing step, to avoid expensive refinement iterations over loops [23].

Bounded model checking of programs. Efficient SAT-based techniques have been used for bounded model checking (BMC) [5] of programs in tools such as CBMC [7], F-Soft [21], and for scalable summary-based analysis in Saturn [33]. These techniques automatically utilize SAT-based conflict analysis and learning for pruning the search space. However, they suffer in the presence of loops, which require deep unwindings that result in large SAT problems. Furthermore, BMC typically handles all paths up to some bounded length as a single monolithic problem. In contrast, we encode only the control paths as a SAT formula, which is much smaller than a typical BMC formula that encodes unwindings of a program.

Abstract interpretation and path-sensitive analysis. Other approaches to path sensitive analysis include ESP [14], trace partitioning [25], elaborations [31], amongst many others. These techniques employ heuristics to control the trade-off between performing a join operation or a logical disjunction at the merge points in the CFG. However, the join vs. disjunction choice is inferred in our SMPP scheme by the disjunction-based decomposition over control paths.

\section{CONCLUSION}

We have presented the Satisfiability Modulo Path Programs (SMPP) approach to program analysis, which lifts to 
the architecture of SMT solvers to path-sensitive program analysis. We have demonstrated it to be effective in analyzing real-world programs.

\section{REFERENCES}

[1] Gogul Balakrishnan, Sriram Sankaranarayanan, Franjo Ivancic, Ou Wei, and Aarti Gupta. SLR: Path-sensitive analysis through infeasible-path detection and syntactic language refinement. In $S A S$, volume 5079 of $L N C S$, pages 238-254, 2008.

[2] Thomas Ball and Sriram K. Rajamani. The slam toolkit. In $C A V$, volume 2102 of $L N C S$, pages 260-264, 2001.

[3] Dirk Beyer, Thomas A. Henzinger, Ranjit Jhala, and Rupak Majumdar. The software model checker blast. STTT, 9(5-6):505-525, 2007.

[4] Dirk Beyer, Thomas A. Henzinger, Rupak Majumdar, and Andrey Rybalchenko. Path invariants. In PLDI, pages 300-309. ACM, 2007.

[5] Armin Biere, Alessandro Cimatti, Edmund Clarke, and Yunshan Zhu. Symbolic model checking without BDDs. In TACAS, volume 1579 of $L N C S$, pages 193-207, 1999.

[6] Aaron R. Bradley and Zohar Manna. Property-directed incremental invariant generation. Formal Asp. Comput., 20(4-5):379-405, 2008.

[7] Edmund Clarke, Daniel Kroening, and Flavio Lerda. A tool for checking ANSI-C programs. In TACAS, volume 2988 of $L N C S, 2004$.

[8] Edmund M. Clarke, Orna Grumberg, Somesh Jha, Yuan Lu, and Helmut Veith. Counterexample-guided abstraction refinement. In $C A V$ '00, pages 154-169. Springer, 2000.

[9] Thoman H. Cormen, Charles E. Leiserson, and Ronald L. Rivest. Introduction to Algorithms. MIT Press, 1992.

[10] P. Cousot and R. Cousot. Static determination of dynamic properties of programs. In Proc. ISOP'76, pages 106-130. Dunod, Paris, France, 1976.

[11] P. Cousot and R. Cousot. Comparing the Galois connection and widening/narrowing approaches to Abstract interpretation, invited paper. In PLILP '92, volume 631 of LNCS, pages 269-295. Springer, 1992.

[12] Patrick Cousot and Radhia Cousot. Abstract Interpretation: A unified lattice model for static analysis of programs by construction or approximation of fixpoints. In POPL' 'r', pages 238-252, 1977.

[13] Patrick Cousot and Nicholas Halbwachs. Automatic discovery of linear restraints among the variables of a program. In POPL'78, pages 84-97, January 1978.

[14] Manuvir Das, Sorin Lerner, and Mark Seigle. Esp: Path-sensitive program verification in polynomial time. In PLDI, pages 57-68, 2002.

[15] Leonardo Mendonça de Moura and Nikolaj Bjørner. Z3: An efficient SMT solver. In TACAS, volume 4963 of $L N C S$, pages 337-340. Springer, 2008.

[16] Bruno Dutertre and Leonardo Mendonça de Moura. A fast linear-arithmetic solver for dpll(t). In $C A V$, volume 4144 of $L N C S$, pages 81-94. Springer, 2006.

[17] Sussane Graf and Hasan Saidi. Construction of abstract state graphs with PVS. In $C A V^{\prime} 97$, volume 1254 of $L N C S$, pages 72-83, 1997.
[18] Sumit Gulwani, Saurabh Srivastava, and Ramarathnam Venkatesan. Program analysis as constraint solving. In PLDI, pages 281-292, 2008.

[19] Ashutosh Gupta and Andrey Rybalchenko. InvGen: An efficient invariant generator. In $C A V$, volume 5643 of $L N C S$, pages 634-640, 2009.

[20] Matthias Heizmann, Jochen Hoenicke, and Andreas Podelski. Refinement of trace abstraction. In Static Analysis Symposium (SAS'09), volume 5673 of $L N C S$, pages 69-85. Springer, 2009.

[21] Franjo Ivancic, Zijiang Yang, Malay K. Ganai, Aarti Gupta, Ilya Shlyakhter, and Pranav Ashar. F-soft: Software verification platform. In $C A V$, volume 3576 of $L N C S$, pages 301-306. Springer, 2005.

[22] Franjo Ivančić, Sriram Sankaranarayanan, Ilya Shlyakhter, and Aarti Gupta. Buffer overflow analysis using environment refinement. Draft (2009), Available Upon Request.

[23] Himanshu Jain, Franjo Ivančić, Aarti Gupta, Ilya Shlyakhter, and Chao Wang. Using statically computed invariants inside the predicate abstraction and refinement loop. In $C A V^{\prime} 06$, pages 137-151, 2006.

[24] Ranjit Jhala and Rupak Majumdar. Path slicing. In PLDI, pages 38-47, 2005.

[25] Laurent Mauborgne and Xavier Rival. Trace partitioning in abstract interpretation based static analyzers. In ESOP'05, volume 3444 of $L N C S$, pages 5-20, 2005.

[26] Kenneth L. McMillan. Lazy abstraction with interpolants. In $C A V^{\prime} 06$, volume 4144 of $L N C S$, pages 123-136, 2006.

[27] Antoine Miné. A new numerical abstract domain based on difference-bound matrices. In PADO II, volume 2053 of $L N C S$, pages 155-172. Springer, May 2001.

[28] Matthew W. Moskewicz, Conor F. Madigan, Ying Zhao, Lintao Zhang, and Sharad Malik. Chaff: Engineering an efficient sat solver. In $D A C$, pages 530-535. ACM, 2001.

[29] Robert Nieuwenhuis, Albert Oliveras, and Cesare Tinelli. Solving SAT and SAT modulo theories: From an abstract davis-putnam-logemann-loveland procedure to DPLL(T). J. ACM, 53(6):937-977, 2006.

[30] Sriram Sankaranarayanan, Franjo Ivančić, and Aarti Gupta. Program analysis using symbolic ranges. In $S A S$, volume 4634 of $L N C S$, pages 366-383, 2007.

[31] Sriram Sankaranarayanan, Franjo Ivancic, Ilya Shlyakhter, and Aarti Gupta. Static analysis in disjunctive numerical domains. In $S A S$, volume 4134 of $L N C S$, pages 3-17. Springer, 2006.

[32] John Whaley and Monica S. Lam. Cloning-based context-sensitive pointer analysis using binary decision diagrams. In PLDI'04. ACM Press, June 2004.

[33] Yichen Xie and Alex Aiken. Saturn: A scalable framework for error detection using boolean satisfiability. ACM Trans. Program. Lang. Syst., 29(3), 2007.

[34] Misha Zitser, Richard Lippmann, and Tim Leek. Testing static analysis tools using exploitable buffer overflows from open source code. In Proc. SIGSoft/FSE'04, pages 97-106. ACM, 2004. 